Article

\title{
Polydimethylsiloxane Rods for the Passive Sampling of Pesticides in Surface Waters
}

Azziz Assoumani ${ }^{1, *}$, Christelle Margoum ${ }^{1}$, Yannick Lassalle ${ }^{1,2}$, Bernard Herbreteau ${ }^{2}$, Karine Faure ${ }^{2}$, Marina Coquery ${ }^{1}$ and Jérôme Randon ${ }^{2}$

1 Irstea, UR MALY, 5 rue de la Doua, CS70077, F-69626 Villeurbanne Cedex, France; E-Mails: christelle.margoum@irstea.fr (C.M.); yann.lassalle@gmail.com (Y.L.); marina.coquery@irstea.fr (M.C.)

2 Institut des Sciences Analytiques, UMR 5280, Université de Lyon, Université Claude Bernard Lyon 1, 43 Boulevard du 11 Novembre 1918, F-69622 Villeurbanne Cedex, France;

E-Mails: bernard.herbreteau@univ-lyon1.fr (B.H.); karine.faure@univ-lyon1.fr (K.F.); jerome.randon@univ-lyon1.fr (J.R.)

* Author to whom correspondence should be addressed; E-Mail: azziz.assoumani@irstea.fr; Tel.: +334-72-20-10-86; Fax: +334-78-47-78-75.

Received: 19 June 2013; in revised form: 19 July 2013 / Accepted: 27 August 2013 /

Published: 11 September 2013

Abstract: In this work, the low cost synthesis of polydimethylsiloxane (PDMS) rods is described, and the performances of this new passive sampling device (in laboratory and in situ) are compared to the passive stir bar sorptive extraction (SBSE) for the monitoring of pesticides from different classes (herbicides, insecticides and fungicides) in surface waters. The influence of synthesis parameters of PDMS rods (i.e., heating temperature, heating time and relative amount of curing agent) were assessed regarding their efficiency for the extraction of the target pesticides through a Hadamard's experimental design. This allowed the determination of the effect of the three parameters on the sorption of pesticides within four experiments. Thus, specific conditions were selected for the synthesis of the PDMS rods (heating at $80{ }^{\circ} \mathrm{C}$ for $2 \mathrm{~h}$ with $10 \%$ of curing agent). Laboratory experiments led to similar to lower extraction recovery in the PDMS rods in comparison with passive SBSE, depending on the pesticide. The in situ application demonstrated the efficiency of the PDMS rods for the passive sampling of the target pesticides in river water, although lower amounts of pesticides were recovered in comparison with passive SBSE. So, these very low cost PDMS rods could be used as an alternative to passive SBSE for large-scale monitoring campaigns. 
Keywords: polydimethylsiloxane (PDMS) rods; passive sampling; SBSE; pesticides; surface waters

\section{Introduction}

Passive sampling has recently been developed as an alternative technique to grab sampling and automated sampling for the monitoring of environmental pollutants in surface and ground waters because this technique allows the determination of time-weighted average (TWA) concentrations with less logistical needs and lower analytical cost. Once immersed in the aquatic medium, the passive sampler accumulates chemicals from large amounts of water and the integrative sampling of the dissolved fraction of pollutants over days to weeks leads to low quantification limits for various organic contaminants [1-4]. A passive sampler is typically composed of a receiving phase, where the target pollutants are accumulated, and a membrane, which separates the receiving phase from the medium. Depending on the type of membrane and receiving phase, chemicals with different polarities can be sampled. Several devices have been developed for the monitoring of hydrophobic organic compounds, such as polycyclic aromatic hydrocarbons (PAH) and polychlorinated biphenyls (PCB) [5-9], and also for polar compounds such as pesticides [10,11]. Stir bar sorptive extraction (SBSE) was recently used directly in situ as a membrane-free passive sampling technique (passive SBSE) and its efficiency was demonstrated for the sampling of moderately polar to hydrophobic pesticides [12]. An SBSE stir bar (Twister ${ }^{\mathbb{R}}$ ) is composed of a magnet enclosed in a glass tube coated with a thick film of polydimethylsiloxane (PDMS) $[13,14]$. After the extraction step, the chemicals sorbed in the PDMS film can be recovered using organic solvents (liquid desorption) or heat (thermal desorption), before analysis by liquid or gas chromatography. However, in case of in situ applications [12], the magnet of the Twister is not necessary because the stir bar does not need to rotate while it is immersed in the medium. Additionally, the cleaning of mineral material from the PDMS phase or the decontamination of organic compounds can be difficult after direct immersion in surface waters and so, cross-contamination could occur if the stir bar is reused for sampling.

Hence, the aim of this work was to develop disposable PDMS rods, with repeatable composition, dimensions and weight, and to assess the performances of this new passive sampling tool for the accumulation of pesticides in surface freshwaters. First, we determined the synthesis conditions for the PDMS rods using a Hadamard's experimental design. Then, we studied PDMS rod sorption capacities for a set of selected pesticides with different physical chemical properties, and compared them to commercial Twisters. Finally, we used PDMS rods in a river located in a French vineyard watershed to assess their performances for the passive sampling of the target pesticides.

\section{Experimental Section}

\subsection{Chemicals and Materials}

From a large range of pesticides usually encountered in the freshwaters of vineyard watersheds [15], we selected a relevant set of six pesticides from three classes (herbicides, insecticides 
and fungicides), covering a broad range of hydrophobicity $\left(2.69<\log K_{\text {ow }}<4.31\right)$ : 3,4-dichloroaniline (metabolite of diuron), spiroxamine, procymidon, fenitrothion, diflufenican, and chlorpyrifos-methyl. The selected pesticides (purity $\geq 92.5 \%$ ) were provided by Dr. Ehrenstorfer (Augsburg, Germany). Diuron-d6, used as internal standard, was also provided by Dr. Ehrenstorfer (purity =98.5\%). Sodium chloride $\mathrm{NaCl}$ (purity $=99 \%-100.5 \%$ ) was purchased from VWR (Strasbourg, France). Dichloromethane for pesticide residue analysis, and liquid chromatography-mass spectrometry (LC-MS) grade acetonitrile and methanol were purchased from VWR (Strasbourg, France). Formic acid (purity $=98 \%$ ) for LC-MS analysis was provided by Fischer Bioblock (Illkirch, France). Ultrapure water was produced by a MilliQ water purification system equipped with a LC-Pak cartridge and purchased from Millipore (Billerica, MA, USA). Twisters (20 $\mathrm{mm} \times 1$-mm thick PDMS film, with a surface area of $2.14 \mathrm{~cm}^{2}$ and a volume of $126 \mu \mathrm{L}$ ) were purchased from Gerstel (Mülheim a/d Ruhr, Germany). A Sylgard 184 kit (Dow Corning, Midland, MI, USA), composed of a PDMS base and a curing agent (cross-linker) was used for the preparation of the PDMS rods. A Shimadzu 17A gas chromatography oven (Marne-la-Vallée, France) was used for the curing of the PDMS.

\subsection{Experimental Design for the Synthesis of the PDMS Rods}

For the preparation of the PDMS rods, the desired amounts of viscous PDMS and curing agent from the Sylgard 184 kit were mixed vigorously in a beaker for 2 min. Then, the beaker was placed in a vacuum chamber for $30 \mathrm{~min}$ in order to remove air bubbles from the mixture. Twenty grams of the degassed mixture was poured into a 9-cm plastic Petri dish, in order to form a 3-mm thick PDMS sheet. The Petri dish was placed horizontally in a GC oven and the heating leads to the cross-linking of PDMS polymer chains and hardens the material. In order to obtain dimensions similar to those of the Twisters, the PDMS sheet was carefully cut into rods of $2 \mathrm{~cm} \times 3 \mathrm{~mm} \times 3 \mathrm{~mm}$ (i.e., surface area of $2.6 \mathrm{~cm}^{2}$ and volume of $180 \mu \mathrm{L}$ ). The PDMS rods were weighted and, to ensure homogeneity, only those with masses ranging from $170 \mathrm{mg}$ to $180 \mathrm{mg}$ were kept for the sorption study. Finally, the PDMS rods were rinsed three times with $50 \mathrm{~mL}$ methanol/dichloromethane (50:50, v/v) for $15 \mathrm{~min}$ using sonication, were gently dried with Kimwipes ${ }^{\circledR}$ precision paper and placed at $50{ }^{\circ} \mathrm{C}$ for one hour.

Relative amount of curing agent, heating temperature and heating time of the curing reaction are the main parameters influencing the mechanical properties of PDMS [16]. In our study, a Hadamard's experimental design was used to investigate if these parameters also have an influence on the sorption performances of the resulting PDMS rods. Two levels were used for the three selected parameters: $70{ }^{\circ} \mathrm{C}$ and $90{ }^{\circ} \mathrm{C}$ for heating temperature, $70 \mathrm{~min}$ and $90 \mathrm{~min}$ for heating time, and $8 \%$ and $12 \%$ for the relative amount of curing agent. These ranges include the usual synthesis conditions, which are $80{ }^{\circ} \mathrm{C}$ for $2 \mathrm{~h}$, with $10 \%$ of curing agent [16]. With the assumption that these parameters are independent, such experimental design allowed us to determine their effect on the sorption of pesticides within four experiments (Table 1). So, four different syntheses were realized (i.e., PDMS_1, PDMS_2, PDMS_3 and PDMS_4). Then, laboratory sorption experiments were performed to compare the sorption performances of these four types of PDMS rods. 
Table 1. Description of the Hadamard's matrix of experiments with two levels for three parameters tested for polydimethylsiloxane (PDMS) synthesis (heating temperature, heating time and curing agent relative amount).

\begin{tabular}{cccc}
\hline PDMS Type & Temperature $\left({ }^{\circ} \mathbf{C}\right)$ & Time $(\mathbf{h})$ & Curing Agent $(\%)$ \\
\hline PDMS_1 & 70 & 3 & 8 \\
PDMS_2 & 70 & 1 & 12 \\
PDMS_3 & 90 & 3 & 12 \\
PDMS_4 & 90 & 1 & 8 \\
\hline
\end{tabular}

\subsection{Laboratory Sorption Experiments}

\subsubsection{Comparison of the Extraction Recoveries of the Four Types of PDMS Rods}

Batch sorption tests with the four types of PDMS rods were performed in order to assess their sorption performances and to determine the best synthesis conditions. The four types of PDMS rods and Twisters, used as reference, were exposed in triplicates to the same pesticide solution. The extraction recoveries were determined with the ratio of the mass of analyte in the extraction phase over the initial mass of analyte in the water sample.

In our experimental conditions (i.e., stirring for $3 \mathrm{~h}$ ), we previously demonstrated that equilibrium state is reached for Twisters [17]. The PDMS rods and the Twisters were placed in 30-mL amber glass vials containing $20 \mathrm{~mL}$ of a water solution spiked with the selected pesticides at different concentrations, depending on the analytical sensitivity of the compound $\left(0.5 \mu \mathrm{g} \mathrm{L}^{-1}\right.$ for spiroxamine, $2 \mu \mathrm{g} \mathrm{L}^{-1}$ for diflufenican, $8 \mu \mathrm{g} \mathrm{L}^{-1}$ for chlorpyrifos-methyl, $10 \mu \mathrm{g} \mathrm{L}^{-1}$ for 3,4-dichloroaniline, and $20 \mu \mathrm{g} \mathrm{L}^{-1}$ for fenitrothion and procymidon) and $2.0 \mathrm{~g}$ of $\mathrm{NaCl}$ under magnetic stirring at $700 \mathrm{rpm}$ with a Variomag 15-position magnetic stirrer purchased from Thermo (Waltham, MA, USA).

Prior to use, PDMS rods and commercial Twisters were conditioned using a method previously developed for SBSE [17]. Briefly, they were placed overnight at $50{ }^{\circ} \mathrm{C}$, then they were rinsed with $10 \mathrm{~mL}$ methanol/dichloromethane $(50: 50, \mathrm{v} / \mathrm{v})$ for $30 \mathrm{~min}$ by sonication, gently dried with Kimwipes ${ }^{\circledR}$ precision paper and placed at $50{ }^{\circ} \mathrm{C}$ for one hour.

\subsubsection{Sorption Capacity Tests}

Extractions of water solutions spiked with the selected pesticides were carried out with both PDMS rod (synthesized according to the parameters selected following the experimental design) and Twister. The sorption capacity tests were performed as described in Section 2.3.1 but with different pesticide concentration levels. Seven PDMS rods and seven Twisters were placed in $30 \mathrm{~mL}$ amber glass vials containing $20 \mathrm{~mL}$ of a water solution spiked with the selected pesticides at increasing concentrations, ranging from 0.05 to $2.5 \mu \mathrm{g} \mathrm{L} \mathrm{L}^{-1}$ for spiroxamine, from 0.2 to $10 \mu \mathrm{g} \mathrm{L}^{-1}$ for diflufenican, from 0.8 to $40 \mu \mathrm{g} \mathrm{L}^{-1}$ for chlorpyrifos-methyl, from 1 to $50 \mu \mathrm{g} \mathrm{L}^{-1}$ for 3,4-dichloroaniline, from 2 to $100 \mu \mathrm{g} \mathrm{L}^{-1}$ for fenitrothion, and from 4 to $100 \mu \mathrm{g} \mathrm{L}^{-1}$ for procymidon. The PDMS rods and the Twisters were recovered after $5 \mathrm{~h}$ of stirring. After the extraction step, PDMS rods and Twisters were gently rinsed with ultrapure water, dried with Kimwipes ${ }^{\circledR}$ precision paper, then placed overnight at $-18{ }^{\circ} \mathrm{C}$ before chemical analysis. 


\subsection{Field Experiments}

Both PDMS rods and Twisters were deployed at two sites in the Morcille River, a small river of a French vineyard watershed located $70 \mathrm{~km}$ north of Lyon: an intermediate site on the river and a downstream site. Details of the sampling sites on the Morcille River are reported elsewhere [15]. Both devices were placed in deployment bags made of plastic mesh, to protect them from small debris, while directly exposing the sorption phases to the aquatic medium (i.e., no membrane used). The passive samplers were placed in triplicate into a rigid plastic container with holes for a two-week exposure period. Field blanks were systematically used for both tools. After the exposition period, PDMS rods and Twisters were brought back to the laboratory in cooled containers for chemical analysis.

\subsection{Chemical Analysis}

Before chemical analysis, pesticides were desorbed from PDMS rods and Twisters according to an analytical method published elsewhere [17]. Briefly, after storage overnight at $-18^{\circ} \mathrm{C}$, Twisters were placed in $200 \mu \mathrm{L}$ of methanol/acetonitrile (50:50, v/v), and pesticides were desorbed under sonication for $15 \mathrm{~min}$. Then, $150 \mu \mathrm{L}$ of ultrapure water and $10 \mu \mathrm{L}$ of diuron-d6 at $200 \mu \mathrm{g} \mathrm{L}^{-1}$ in acetone, were added to $40 \mu \mathrm{L}$ of the desorbate to constitute the extracted sample for analysis by liquid chromatography coupled to tandem mass spectrometry (LC-MS/MS) [17].

The analyses of the extracts were performed using a LC 1100 Series apparatus from Agilent (Massy, France) coupled with a MS triple quadrupole API4000 from AB SCIEX (Les Ulis, France), equipped with an electrospray ionization source (ESI) that was operated in the positive ionization mode. An Atlantis T3 column $\left(2.1 \mathrm{~mm} \times 100 \mathrm{~mm} ; d_{\mathrm{p}}=3 \mu \mathrm{m}\right)$, purchased from Waters (St Quentin-en-Yvelines, France) was used for the chromatographic separation of the analytes. Acetonitrile and ultrapure water both with formic acid $(0.1 \%)$ were used in an analytical gradient (from 10\% to $90 \%$ acetonitrile) of

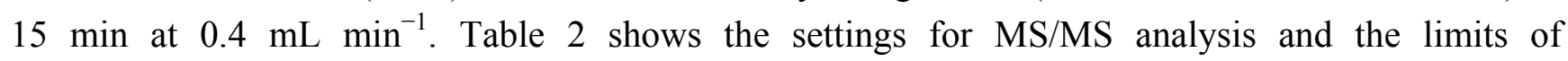
quantification (LQ) of the selected pesticides.

Table 2. Multiple reaction monitoring conditions for $\mathrm{MS} / \mathrm{MS}$ analysis and limits of quantification of the selected pesticides (from Margoum et al. [17]).

\begin{tabular}{|c|c|c|c|c|c|c|c|}
\hline Compound & $\log K_{\text {ow }}$ & $\begin{array}{c}\text { Precursor } \\
\text { Ion }(\mathbf{m} / \mathbf{z})\end{array}$ & $\begin{array}{l}\text { Declustering } \\
\text { Potential (V) }\end{array}$ & $\begin{array}{c}\text { Product } \\
\text { Ions }(\mathbf{m} / \mathbf{z})\end{array}$ & $\begin{array}{c}\text { Collision } \\
\text { Energy (V) }\end{array}$ & $\begin{array}{c}\text { Collision Cell } \\
\text { Exit potential (V) }\end{array}$ & $\begin{array}{c}\mathbf{L Q} \\
\left(\mu \mathrm{g} \mathrm{L}^{-1}\right)\end{array}$ \\
\hline 3,4-dichloroaniline & 2.69 & 162.0 & 51 & $127.0 ; 74.0$ & $31 ; 73$ & $24 ; 14$ & 0.05 \\
\hline Spiroxamine & 2.89 & 297.9 & 51 & $144.3 ; 100.1$ & $31 ; 45$ & $8 ; 18$ & 0.02 \\
\hline Procymidon & 3.08 & 284.0 & 76 & $256.0 ;-$ & $25 ;-$ & $46 ;-$ & 0.20 \\
\hline Fenitrothion & 3.32 & 277.9 & 71 & $124.8 ; 109.0$ & $29 ; 25$ & $22 ; 16$ & 0.50 \\
\hline Diflufenican & 4.20 & 395.0 & 86 & $266.0 ; 246.0$ & $35 ; 47$ & $28 ; 40$ & 0.20 \\
\hline Chlorpyrifos-methyl $^{\text {a }}$ & 4.31 & 322.0 & 71 & $125.0 ; 190.0$ & $29 ; 23$ & $22 ; 54$ & 0.05 \\
\hline
\end{tabular}

Note: ${ }^{a}$ Not presented in the analytical method published by Margoum et al. [17], it was added to the method and validated afterwards. 


\section{Water-PDMS Extraction of Solutes}

Sorptive extraction is a sampling technique governed by diffusion of the analytes from the bulk solution to the PDMS phase. The efficiency of the extraction of the analytes from the aqueous samples into the PDMS extraction phase relies on the equilibrium regime, and the sorption process is essentially a liquid-liquid partition [14].

The partitioning coefficient of the analytes between the extraction phase and the aqueous phase $K_{\text {sw }}$ (adimensional) is linked to the concentration of the analytes in these two phases [18,19] as follows [Equation (1)]:

$$
K_{\mathrm{sw}}=\frac{C_{\mathrm{s}}}{C_{\mathrm{w}}}=\frac{m_{\mathrm{s}}}{m_{\mathrm{w}}} \times \frac{V_{\mathrm{w}}}{V_{\mathrm{s}}}=\frac{m_{\mathrm{s}}}{m_{\mathrm{w}}} \times \beta
$$

where $C_{\mathrm{w}}\left(\mu \mathrm{g} \mathrm{L}^{-1}\right)$ is the concentration of analyte in the water sample at equilibrium; $C_{\mathrm{s}}\left(\mu \mathrm{g} \mathrm{L}^{-1}\right)$ is the concentration of analyte in the extraction phase at equilibrium; $m_{\mathrm{w}}(\mu \mathrm{g})$ is the mass of analyte remaining in the water sample; $m_{\mathrm{s}}(\mu \mathrm{g})$ is the mass of analyte in the extraction phase; $V_{\mathrm{w}}(\mathrm{L})$ is the volume of water sample; $V_{\mathrm{s}}(\mathrm{L})$ is the volume of the extraction phase; $\beta$ (adimensional) is the phase ratio. Several studies have correlated the partitioning coefficient of analytes $K_{\mathrm{sw}}$ with their octanol-water distribution coefficient $\left(K_{\text {ow }}\right)[13,20-24]$.

The extraction recovery $\eta(\%)$ is expressed as the ratio of the mass of analyte in the extraction phase $\left(m_{\mathrm{s}}\right)$ over the initial mass of analyte in the water sample $\left(m_{0}=m_{\mathrm{s}}+m_{\mathrm{W}}\right)$. It is determined by the partitioning coefficient $K_{\mathrm{sw}}$ and by the phase ratio $\beta$, as described in Equation (2).

$$
\eta=\frac{m_{\mathrm{s}}}{m_{0}} \times 100=\frac{K_{\mathrm{sw}} / \beta}{1+\left(K_{\mathrm{sw}} / \beta\right)} \times 100
$$

From Equation (2), it is easily deduced that the extraction recovery increases with $K_{\mathrm{sw}}$. With the assumption that $K_{\mathrm{sw}}$ is correlated to the octanol-water distribution coefficient $K_{\mathrm{ow}}$, the extraction recovery on PDMS, in general, increases with increasing hydrophobicity. Moreover, the phase ratio $\beta$ can also affect the extraction recovery [24].

\section{Results and Discussion}

\subsection{Laboratory Experiments}

\subsubsection{Optimization of the Curing Conditions for PDMS Synthesis}

Figure 1 shows the extraction recovery of the target pesticides sorted by increasing hydrophobicity for the four types of synthesized PDMS rods and for Twisters. Good repeatability of extraction performances $(n=3)$ was generally obtained for the tested pesticides. Relative standard deviations (RSD) of the extraction recovery of the pesticides were lower than 25\% for the PDMS rods, except for spiroxamine in PDMS_3 rod and PDMS_4 rod (38\% and 31\%, respectively).

As we expected from the hydrophobic nature of PDMS, the extraction recoveries for PDMS rods and Twisters increased with the hydrophobicity of the target pesticides. The extraction recoveries ranged from 9\% for 3,4-dichloroaniline in PDMS_3 rod to 64\% for chlorpyrifos-methyl in PDMS_2 rod. The extraction recovery of spiroxamine was four times larger than that of 3,4-dichloroaniline 
although their $\log K_{\text {ow }}$ values were relatively close. Moreover, the extraction recoveries of the most three hydrophobic pesticides were about $60 \%$.

Figure 1. Recovery for the extraction of the target pesticides by the four types of synthesized polydimethylsiloxane (PDMS) rods and Twisters, at $700 \mathrm{rpm}$ for $3 \mathrm{~h}$. Numbers in brackets on the $x$ axis are the octanol-water partitioning coefficients of the target pesticides $\left(\log K_{\text {ow }}\right)$, sorted by increasing values. Errors bars represent \pm standard deviation $(n=3)$.

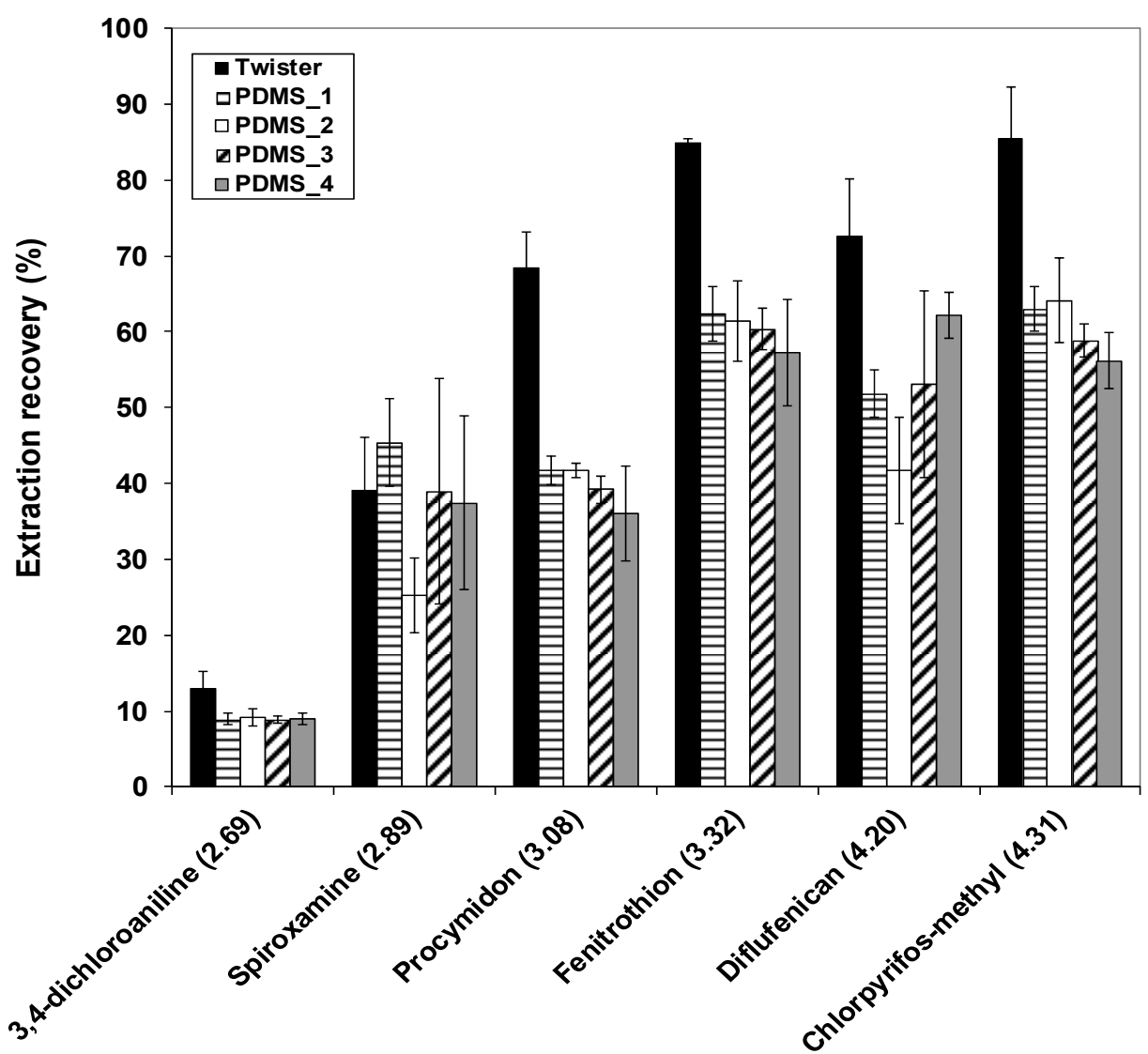

Similar to lower extraction recoveries of the target pesticides were obtained for the four types of PDMS rods in comparison with the Twisters, depending on the pesticide (Figure 1). The extraction recoveries of spiroxamine were similar for the PDMS rods and Twisters. For 3,4-dichloroaniline, the four types of PDMS rods yielded slightly lower recoveries than Twisters, although the recoveries of this pesticide were low for the two devices. For fenitrothion, diflufenican and chlorpyrifos-methyl, lower recoveries were obtained for the four types of PDMS rods in comparison with Twisters but they yielded about $60 \%$ with good repeatability. Further, for procymidon, the extraction recovery of ranged from $36 \%$ to $42 \%$ for the four types of PDMS rods, whereas Twisters achieved a $69 \%$ extraction recovery. The lower amounts of pesticides extracted by PDMS rods was not caused by different kinetics, as kinetic tests confirmed that extraction equilibrium was reach for both devices after $3 \mathrm{~h}$ of stirring (data shown in Appendix). So, the partitioning coefficient of the target pesticides (except spiroxamine) between the PDMS rods and the water sample ( $K_{\text {PDMS-w }}$ ) might be lower than that for the Twisters $\left(K_{\text {Twister-w }}\right)$ because of chemical differences in the polymer structures. 
Comparing the behavior of the different types of PDMS for all tested pesticides, an ANOVA ( $\alpha=0.05$ ) confirmed that the recoveries obtained for the four types of PDMS rods were not significantly different (data not shown). So we assumed that the synthesis parameters had no significant effect on the sorption of pesticides within the ranges studied. Therefore, for the rest of the study, PDMS rods were synthesized at the central point of the experimental design space, i.e., at $80{ }^{\circ} \mathrm{C}$ for $2 \mathrm{~h}$, with $10 \%$ of curing agent.

\subsubsection{Sorption Capacity Tests}

In Figure 2, the results of the sorption capacity tests are presented as sorption isotherms (sorbed mass/phase volume $=\mathrm{f}$ (pesticide concentration in water at equilibrium)) obtained for chlorpyrifos-methyl (a) (initial concentrations ranging from 0.8 to $40 \mu \mathrm{g} \mathrm{L}^{-1}$ ); and spiroxamine (b) (initial concentrations ranging from 0.05 to $2.5 \mu \mathrm{g} \mathrm{L}^{-1}$ ) extracted by PDMS rods and Twisters. Over the investigated pesticide concentration ranges, we did not observe any saturation of neither the PDMS rods nor the Twisters, since satisfactory linearity $\left(R^{2}>0.88\right)$ was obtained for all compounds extracted by the two devices (data not shown).

Figure 2. Sorption isotherms of (a) chlorpyrifos-methyl and (b) spiroxamine on PDMS rods (phase volume $=180 \mu \mathrm{L}$ ) and Twisters (phase volume $=126 \mu \mathrm{L}$ ). Extraction at $700 \mathrm{rpm}$ for $5 \mathrm{~h}$.
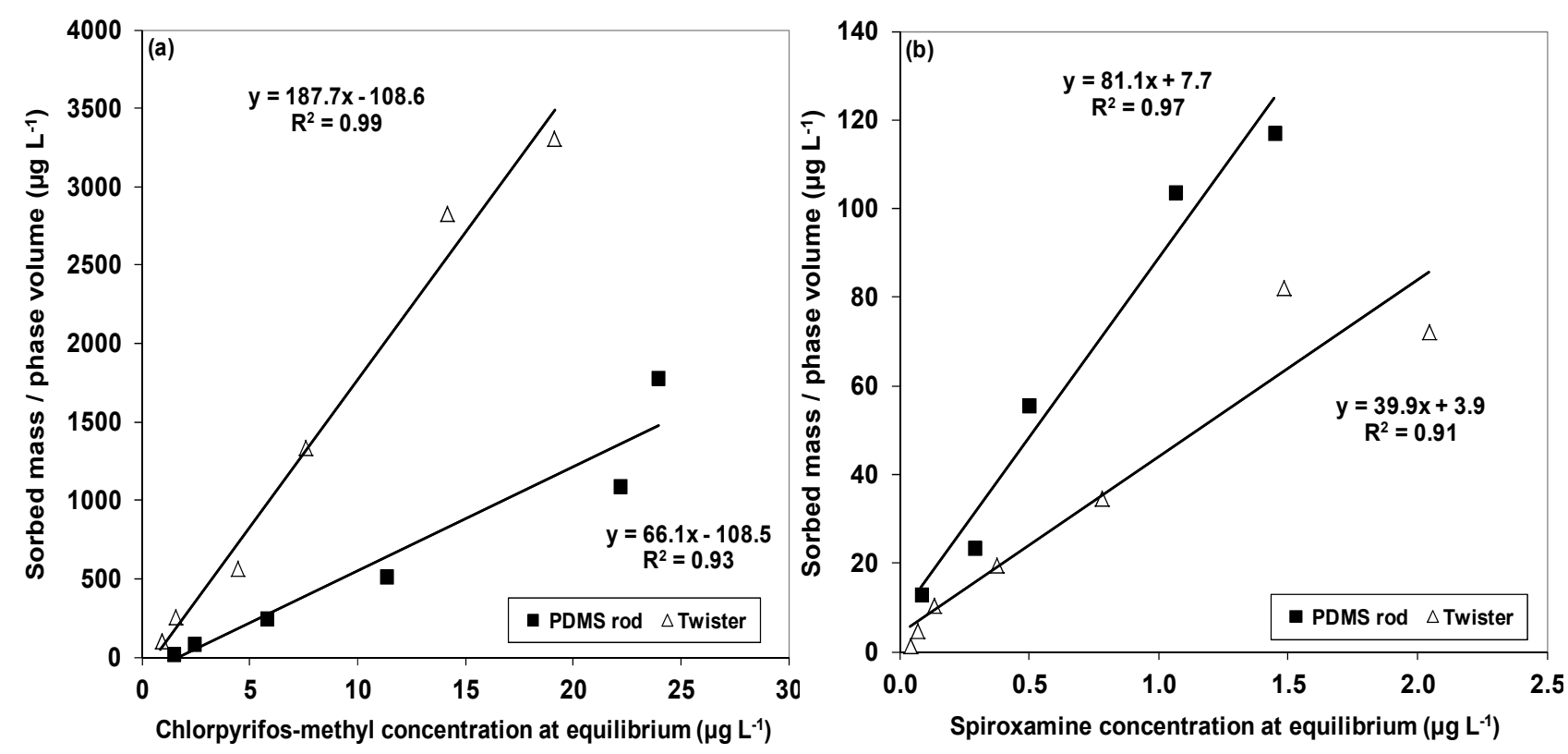

The slope of the sorption isotherms is a useful way to determine the sampler-water sample partitioning coefficients ( $K_{\text {PDMS-w }}$ or $K_{\text {Twister-w }}$ ) [Equation (1)]. The slope of the regression line relative to the PDMS rods for chlorpyrifos-methyl was only $35 \%$ of the slope observed for Twisters (Figure 2a) and the same trend was observed for 3,4-dichloroaniline (70\%), diflufenican (77\%), fenitrothion (36\%), and procymidon (29\%). The opposite was observed for spiroxamine, as the slope of the regression line relative to the PDMS rods was $200 \%$ of the slope observed for Twisters (Figure $2 \mathrm{~b}$ ). Therefore, $K_{\text {PDMS-w }}$ are lower than $K_{\text {Twister-w }}$ for chlorpyrifos-methyl, 3,4-dichloroaniline, diflufenican, fenitrothion and procymidon; so the equilibrium concentrations of these pesticides in 
PDMS rods will always be lower than those in Twisters. For more hydrophobic compounds $\left(\log K_{\mathrm{ow}}>5\right)$, $K_{\text {PDMS-w }}$ and $K_{\text {Twister-w }}$ might be closer, so that the PDMS rods could achieve similar sorption capacity as Twisters.

Both PDMS rods and Twisters are composed of polydimethylsiloxane, but it is very likely that the cross-links of the two polymers are different. Indeed, Ter Laak et al. [25] suggested that differences in cross-linking and polymer length could have an effect on the solubility of hydrophobic chemicals in

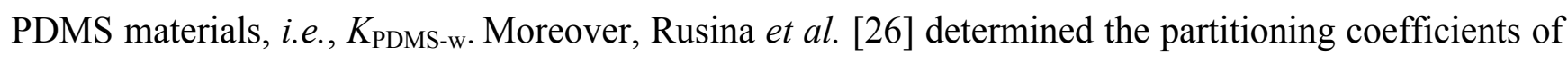
four PAH in several polymers, including PDMS. They observed differences (up to 25\%) in the equilibrium concentrations of the target compounds in PDMS and other silicone materials.

\subsection{Field Experiments}

Figure 3 shows the amounts of spiroxamine, procymidon and diflufenican sorbed in the PDMS rods and Twisters exposed in the Morcille River for two weeks.

Figure 3. Comparison of the amounts of pesticides accumulated in Twisters and PDMS rods exposed in river water for two weeks. "LQ" is the quantification limit in ng for both devices. "nq" stands for non-quantified, "I" stands for the intermediary sampling site and " $D$ " stands for the downstream sampling site. Numbers in brackets on the $x$ axis are the octanol-water partitioning coefficients of the quantified pesticides $\left(\log K_{\mathrm{ow}}\right)$, sorted by increasing values. Errors bars represent \pm standard deviation $(n=3)$.

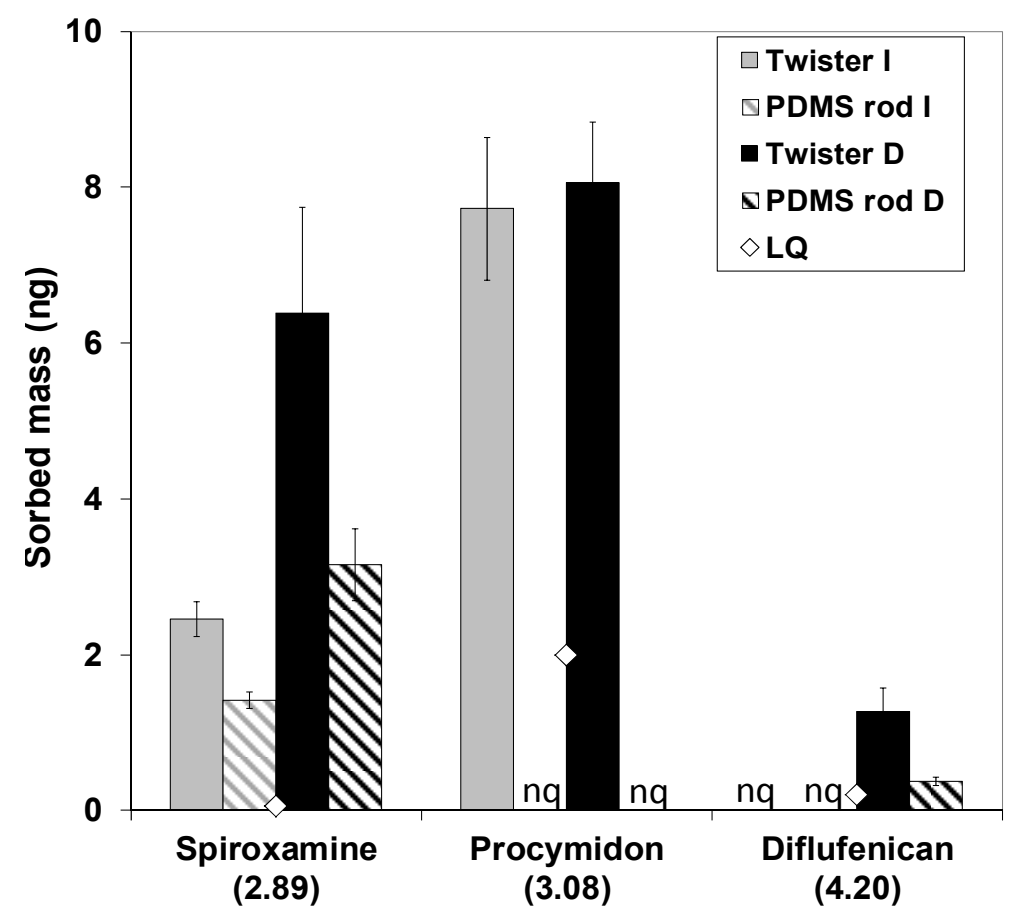

Chlorpyrifos-methyl, 3,4-dichloroaniline and fenitrothion were not detected neither in PDMS rods nor in Twisters. Good repeatability, which includes conditioning, in situ analyte extraction and laboratory chemical analysis, was obtained for both passive sampling devices. RSD ranged from 7\% to $14 \%$ for the PDMS rods and from $9 \%$ to $24 \%$ for the Twisters (for the three detected pesticides, $n=3$ ). The differences in the amounts of spiroxamine, procymidon and diflufenican accumulated in the two 
passive sampling devices were greater than those observed during the laboratory experiments (Section 4.1.1). In addition, procymidon, which is more polar than diflufenican, was not quantified in the PDMS rods, whereas it was accumulated in the Twisters at both sites. A relatively high limit of quantification for this pesticide ( $2 \mathrm{ng}$ ) could be an explication of this observation. Lower amounts of spiroxamine were obtained for PDMS rods in comparison with Twisters, contrary to the larger recovery observed during the laboratory experiments. About $42 \%$ and $51 \%$ less spiroxamine were accumulated in the PDMS rods deployed in the river at the intermediate site and the downstream site, respectively. For spiroxamine, procymidon and diflufenican, such observations could be explained by a fast elimination from the PDMS rods, which could occur if a concentration peak of the analytes has arisen at the beginning of the 14-day exposition. To confirm this hypothesis, the elimination kinetics of the target pesticides need to be investigated for the PDMS rods.

\section{Conclusions}

In this study, we described an easy and fast synthesis of PDMS rods for the passive sampling of moderately hydrophobic to hydrophobic pesticides, and assessed their performances and their applicability in surface freshwaters. After a Hadamard's experimental design, the synthesis conditions were defined and we demonstrated the robustness of the synthesis protocol regarding the sorption properties of the PDMS rods. Laboratory sorption experiments showed similar to lower amounts of the target pesticides quantified in the PDMS rods in comparison with the Twisters used as reference. The lower recoveries observed were due to different partitionings of the pesticides in the two devices. The application of the PDMS rods in a small river of a French vineyard watershed, along with Twisters, confirmed the performances and usefulness of such devices as passive samplers for the target pesticides, although lower amounts of pesticides were recovered. So, PDMS rods represent a promising simple tool for the monitoring of agricultural pesticides in surface freshwaters. Moreover, these PDMS rods could be used for the passive sampling of other types of organic contaminants, especially more hydrophobic ones, for which the sorption capacities might reach those of the Twisters.

This device presents the same advantages as Twisters for passive SBSE, i.e., simple handling, preparation before deployment, in situ deployment and retrieval of the devices, and laboratory sample treatment before chemical analysis. Additionally, the fast and easy, repeatable, and very cost-effective synthesis of the PDMS rods allows to use them in a larger number than Twisters for monitoring campaigns. Moreover, single use of these devices in surface freshwaters prevents possible contamination and advanced clean-up, limiting the use of organic solvents. Further on-going work will focus on the elimination kinetics and the calibration of the PDMS rods in order to allow the determination of TWA concentrations of the target pesticides in surface freshwaters.

\section{Acknowledgments}

The authors thank the French National Agency for Water and Aquatic Environments (ONEMA) and the French National Research Agency (ANR) through the PoToMAC project (ANR 2011 CESA 022 02) for financial support. We also thank C. Guillemain for analytical support, and L. Liger, O. Garcia, and B. Motte for technical and logistical support. 


\section{Conflicts of Interest}

The authors declare no conflict of interest.

\section{References}

1. Namieśnik, J.; Zabiegała, B.; Kot-Wasik, A.; Partyka, M.; Wasik, A. Passive sampling and/or extraction techniques in environmental analysis: A review. Anal. Bioanal. Chem. 2005, 381, 279-301.

2. Vrana, B.; Mills, G.; Allan, I.; Dominiak, E.; Svensson, K.; Knutsson, J.; Morrison, G.; Greenwood, R. Passive sampling techniques for monitoring pollutants in water. TrAC Trends Anal. Chem. 2005, 24, 845-868.

3. Huckins, J.N.; Petty, J.D.; Booij, K. Monitors of Organic Chemicals in the Environment: Semipermeable Membrane Devices, 1st ed.; Springer Science + Business Media: New York, NY, USA, 2006.

4. Greenwood, R.; Mills, G.; Vrana, B. Comprehensive Analytical Chemistry Volume 48: Passive Sampling Techniques in Environmental Monitoring, 1st ed.; Elsevier Science: Amsterdam, The Netherlands, 2007.

5. Huckins, J.N.; Tubergen, M.W.; Manuweera, G.K. Semipermeable membrane devices containing model lipid: A new approach to monitoring the bioavailability of lipophilic contaminants and estimating their bioconcentration potential. Chemosphere 1990, 20, 533-552.

6. Huckins, J.N.; Manuweera, G.K.; Petty, J.D.; Mackay, D.; Lebo, J.A. Lipid-containing semipermeable membrane devices for monitoring organic contaminants in water. Environ. Sci. Technol. 1993, 27, 2489-2496.

7. Vrana, B.; Popp, P.; Paschke, A.; Schüürmann, G. Membrane-Enclosed Sorptive Coating. An Integrative Passive Sampler for Monitoring Organic Contaminants in Water. Anal. Chem. 2001, 73, 5191-5200.

8. Vrana, B.; Paschke, A.; Popp, P. Calibration and field performance of membrane-enclosed sorptive coating for integrative passive sampling of persistent organic pollutants in water. Environ. Pollut. 2006, 144, 296-307.

9. Paschke, A.; Schwab, K.; Brümmer, J.; Schüürmann, G.; Paschke, H.; Popp, P. Rapid semi-continuous calibration and field test of membrane-enclosed silicone collector as passive water sampler. J. Chromatogr. A 2006, 1124, 187-195.

10. Alvarez, D.A.; Petty, J.D.; Huckins, J.N.; Jones-Lepp, T.L.; Getting, D.T.; Goddard, J.P.; Manahan, S.E. Development of a passive, in situ, integrative sampler for hydrophilic organic contaminants in aquatic environments. Environ. Toxicol. Chem. 2004, 23, 1640-1648.

11. Mazzella, N.; Debenest, T.; Delmas, F. Comparison between the polar organic chemical integrative sampler and the solid-phase extraction for estimating herbicide time-weighted average concentrations during a microcosm experiment. Chemosphere 2008, 73, 545-550.

12. Assoumani, A.; Lissalde, S.; Margoum, C.; Mazzella, N.; Coquery, M. In situ application of stir bar sorptive extraction as a passive sampling technique for the monitoring of agricultural pesticides in surface waters. Sci. Total Environ. 2013, 463-464, 829-835. 
13. Baltussen, E.; Sandra, P.; David, F.; Cramers, C. Stir bar sorptive extraction (SBSE), a novel extraction technique for aqueous samples: Theory and principles. J. Microcol. Sep. 1999, 11, 737-747.

14. Prieto, A.; Basauri, O.; Rodil, R.; Usobiaga, A.; Fernandez, L.A.; Etxebarria, N.; Zuloaga, O. Stir-bar sorptive extraction: A view on method optimisation, novel applications, limitations and potential solutions. J. Chromatogr. A 2010, 1217, 2642-2666.

15. Rabiet, M.; Margoum, C.; Gouy, V.; Carluer, N.; Coquery, M. Assessing pesticide concentrations and fluxes in the stream of a small vineyard catchment-Effect of sampling frequency. Environ. Pollut. 2010, 158, 737-748.

16. Liu, M.; Sun, J.; Chen, Q. Influences of heating temperature on mechanical properties of polydimethylsiloxane. Sens. Actuators A 2009, 151, 42-45.

17. Margoum, C.; Guillemain, C.; Yang, X.; Coquery, M. Stir bar sorptive extraction coupled to liquid chromatography-tandem mass spectrometry for the determination of pesticides in water samples: Method validation and measurement uncertainty. Talanta 2013, 116, 1-7.

18. Baltussen, E.; Cramers, C.; Sandra, P. Sorptive sample preparation-A review. Anal. Bioanal. Chem. 2002, 373, 3-22.

19. David, F.; Sandra, P. Stir bar sorptive extraction for trace analysis. J. Chromatogr. A 2007, 1152, 54-69.

20. Baltussen, E.; Sandra, P.; David, F.; Janssen, H.-G.; Cramers, C. Study into the equilibrium mechanism between water and Poly(dimethylsiloxane) for very Apolar solutes: Adsorption or sorption? Anal. Chem. 1999, 71, 5213-5216.

21. Tienpont, B.; David, F.; Bicchi, C.; Sandra, P. High capacity headspace sorptive extraction. J. Microcol. Sep. 2000, 12, 577-584.

22. Popp, P.; Bauer, C.; Wennrich, L. Application of stir bar sorptive extraction in combination with column liquid chromatography for the determination of polycyclic aromatic hydrocarbons in water samples. Anal. Chim. Acta 2001, 436, 1-9.

23. Popp, P.; Bauer, C.; Hauser, B.; Keil, P.; Wennrich, L. Extraction of polycyclic aromatic hydrocarbons and organochlorine compounds from water: A comparison between solid-phase microextraction and stir bar sorptive extraction. J. Sep. Sci. 2003, 26, 961-967.

24. Bicchi, C.; Cordero, C.; Rubiolo, P.; Sandra, P. Impact of water/PDMS phase ratio, volume of PDMS, and sampling time on Stir Bar Sorptive Extraction (SBSE) recovery of some pesticides with different $\mathrm{K}_{\mathrm{O} / \mathrm{w}}$. J. Sep. Sci. 2003, 26, 1650-1656.

25. Ter Laak, T.L.; Busser, F.J.M.; Hermens, J.L.M. Poly(dimethylsiloxane) as passive sampler material for hydrophobic chemicals: effect of chemical properties and sampler characteristics on partitioning and equilibration times. Anal. Chem. 2008, 80, 3859-3866.

26. Rusina, T.P.; Smedes, F.; Klanova, J.; Booij, K.; Holoubek, I. Polymer selection for passive sampling: A comparison of critical properties. Chemosphere 2007, 68, 1344-1351. 


\section{Appendix}

Figure A1. (a) Sorption kinetics of procymidon (at $5 \mu \mathrm{g} \mathrm{L}{ }^{-1}$ ); (b) diflufenican (at $0.5 \mu \mathrm{g} \mathrm{L}^{-1}$ ); (c) fenitrothion (at $5 \mu \mathrm{g} \mathrm{L}^{-1}$ ); (d) 3,4-dichloroaniline (at $2.5 \mu \mathrm{g} \mathrm{L}^{-1}$ ); (e) chlorpyrifos-methyl (at $2 \mu \mathrm{g} \mathrm{L}^{-1}$ ); and (f) spiroxamine (at $0.125 \mu \mathrm{g} \mathrm{L}^{-1}$ ) for PDMS_5 rods and Twisters. Extraction at $700 \mathrm{rpm}$ for maximum $5 \mathrm{~h}$.

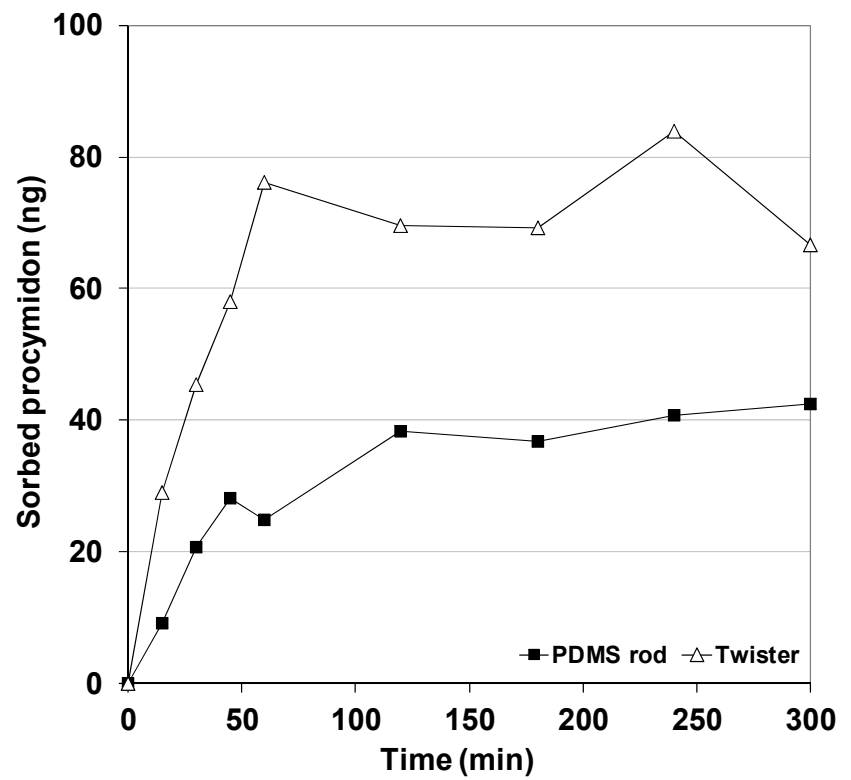

(a)

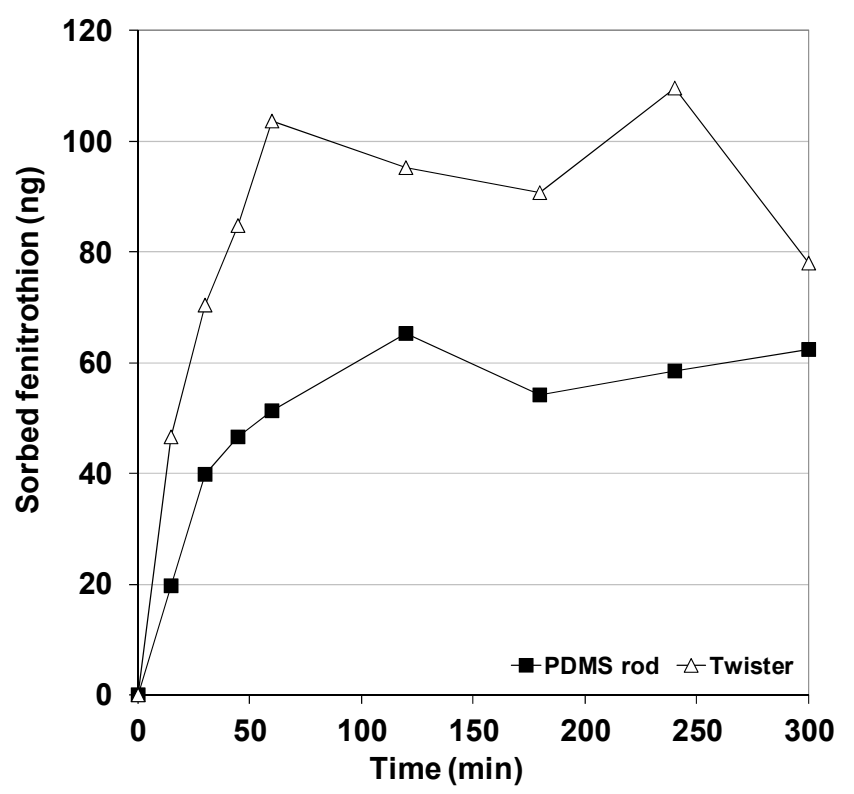

(c)

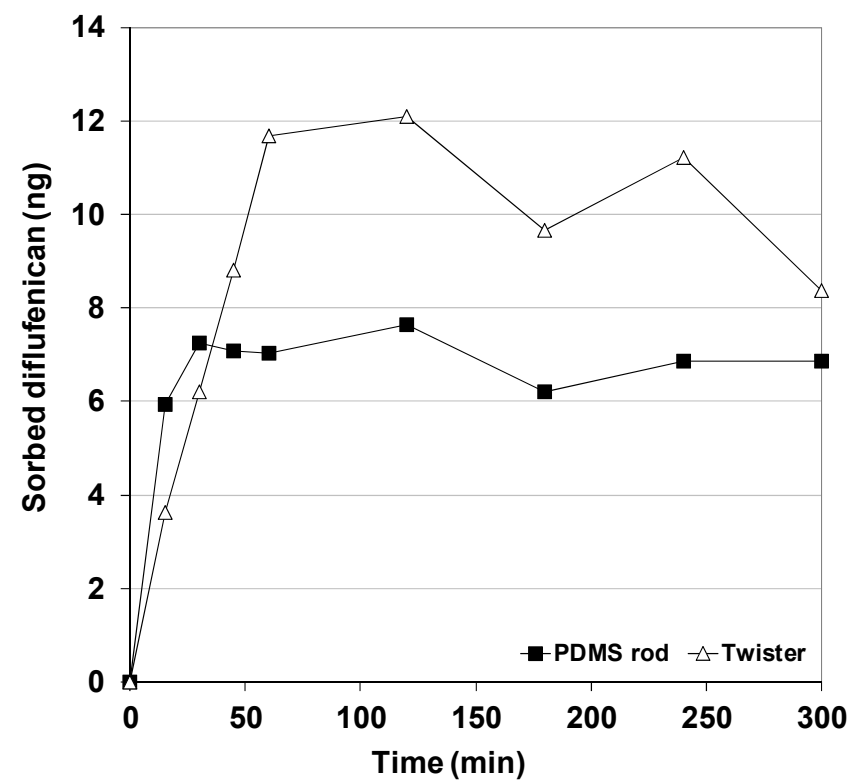

(b)

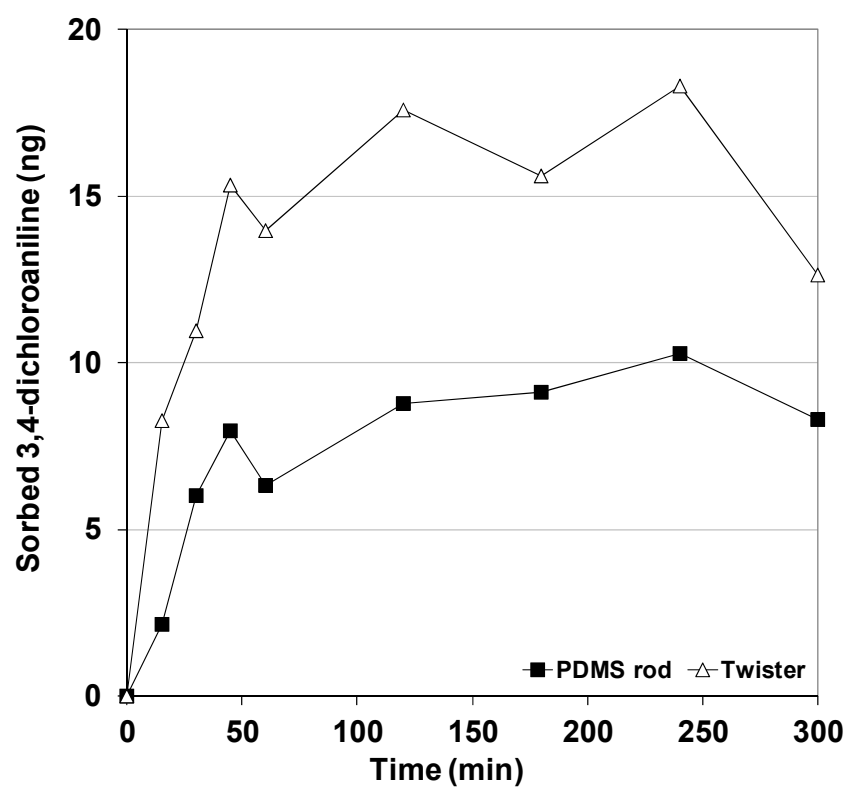

(d) 
Figure A1. Cont.

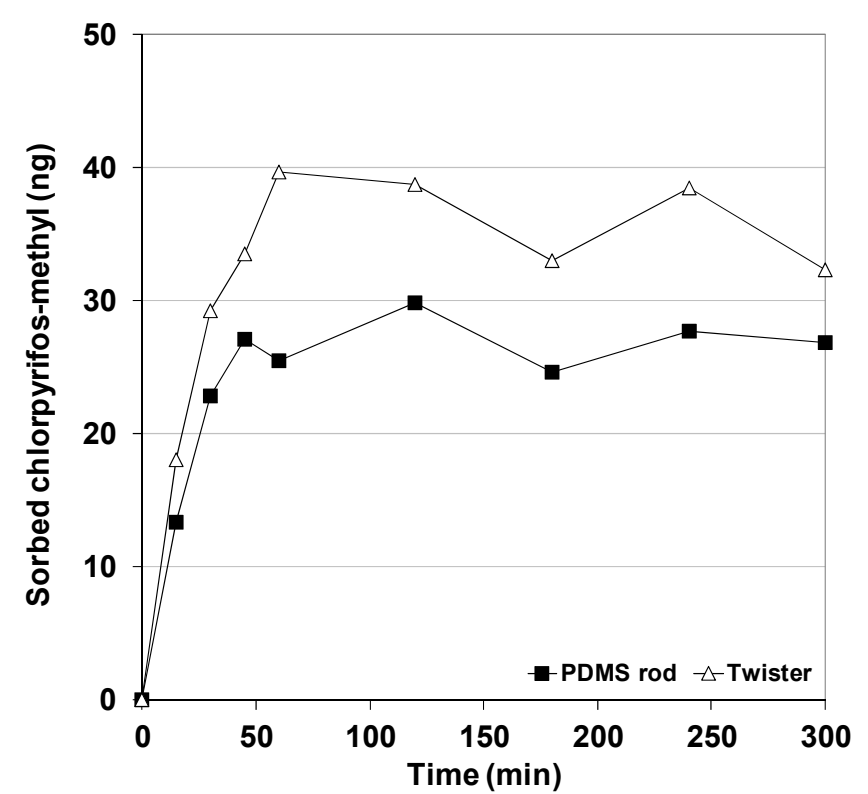

(e)

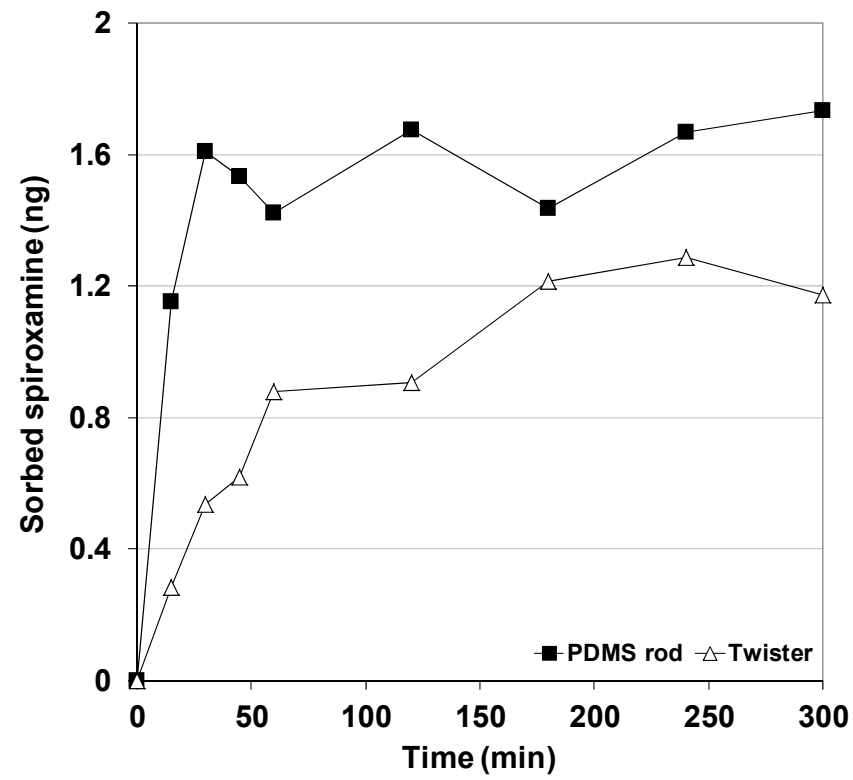

(f)

(C) 2013 by the authors; licensee MDPI, Basel, Switzerland. This article is an open access article distributed under the terms and conditions of the Creative Commons Attribution license (http://creativecommons.org/licenses/by/3.0/). 